\title{
Радиационные термоэлементы на основе теллурида висмута, получаемые методом импульсного лазерного осаждения
}

\author{
(C) А.Е. Шупенев ${ }^{1}$, И.С. Коршунов ${ }^{1}$, А.С. Ильин ${ }^{2}$, А.С. Осипков ${ }^{1}$, А.Г. Григорьянц ${ }^{1}$ \\ ${ }^{1}$ Московский государственный технический университет им. Н.Э. Баумана, \\ 105005 Москва, Россия \\ ${ }^{2}$ Институт теоретической и прикладной электродинамики Российской академии наук, \\ 125412 Москва, Россия \\ E-mail: ash@bmstu.ru
}

Поступила в Редакцию 7 февраля 2019 г.

В окончательной редакции 10 февраля 2019 г.

Принята к публикации 14 февраля 2019 г.

\begin{abstract}
Радиационные термоэлементы являются структурной единицей сенсоров, используемых при измерении энергетических параметров различного излучения в диапазоне длин волн от 0.1 до 100 мкм. В настоящей работе исследуется вопрос перспективности использования пленок $p$ - $\mathrm{Bi}_{0.5} \mathrm{Sb}_{1.5} \mathrm{Te}_{3}$ и $n$ - $\mathrm{Bi}_{2} \mathrm{Te}_{2.7} \mathrm{Se}_{0.3} \quad$ в радиационных термоэлементах на различных подложках, полученных методом импульсного лазерного осаждения. Теплофизический расчет и экспериментальные исследования опытных образцов показали, что с использованием полиимидных подложек можно обеспечить коэффициент преобразования около 1 В/Вт для приемной площадки диаметром 16 мм, при этом постоянная времени составит около $10 \mathrm{c.}$
\end{abstract}

DOI: $10.21883 /$ FTP.2019.06.47722.31

\section{1. Введение}

Тонкие пленки термоэлектрических материалов используются при создании термоэлементов в радиометрии $[1,2]$, микрокалориметрии [3-5], для измерения концентрации газов $[6,7]$, в том числе и на гибких подложках $[8,9]$. Широкодиапазонные приемники излучения (ПИ) на основе радиационных термоэлементов (,thermopile“) используются для измерения энергетических характеристик лазерного излучения [10,11], тепловых потоков [12,13], в MEMS-измерительных микросистемах [14], в космических аппаратах $[15,16]$ и др.

Эффективность термоэлектрических изделий, в том числе пленочных, во многом зависит от эффективности применяемых термоэлектрических материалов [17,18]. Наиболее эффективные низкотемпературные материалы (от 0 до $200^{\circ} \mathrm{C}$ ) на основе теллурида висмута $[19,20]$ получают в виде тонких пленок различными методами: термическим испарением в вакууме [21,22], магнетронным распылением [23,24], молекулярно-лучевой эпитаксией $[25,26]$, осаждением из газовой фазы $[27,28]$ и др. Ряд работ рассматривает использование двойных сплавов на основе теллурида висмута перспективным для радиационных термоэлементов [29,30]. Метод импульсного лазерного осаждения перспективен для получения высокоэффективных термоэлектрических пленок на основе двойных [31,32] и тройных [33,34] сплавов теллурида висмута благодаря возможности конгруэнтного переноса материала многокомпонентных мишеней [35,36].

Поэтому текущая работа посвящена исследованию перспектив использования радиационных термоэлементов, выполненных из тонких пленок $p$ - $\mathrm{Bi}_{0.5} \mathrm{Sb}_{1.5} \mathrm{Te}_{3}$ и $n-\mathrm{Bi}_{2} \mathrm{Te}_{2.7} \mathrm{Se}_{0.3}$ на различных подложках, получаемых методом импульсного лазерного осаждения.
Конструктивно, чувствительный элемент термоэлектрического приемника излучения состоит из тонкопленочных термопар, сформированных на поверхности подложки вокруг приемной площадки. Принцип работы таких приемников заключается в том, что при поглощении приемной площадкой оптического излучения образуется градиент температур между приемной площадкой и „холодными“ спаями, а полученный в результате термоэлектрического эффекта электрический сигнал фиксируется измерительными приборами [37-39].

\section{2. Теоретическая часть}

В приведенных выше работах по приемникам излучения выведены закономерности, связывающие основные характеристики приемников с теплофизическими свойствами используемых материалов и их конструктивными особенностями. Наиболее общие выражения, на основе которых проведен анализ характеристик предложенных конструкций, приведены в работах $[40,41]$.

Коэффициент преобразования по постоянному потоку $R,[\mathrm{~B} / \mathrm{BT}]$ :

$$
R=\varepsilon \cdot \alpha \cdot n / Q_{\Sigma},
$$

где $\varepsilon-$ коэффициент поглощения приемной площадки, $\alpha$ - термоэдс термопары $[\mathrm{B} / \mathrm{K}], n-$ число термопар, $Q_{\Sigma}-$ суммарная эквивалентная тепловая проводимость элементов чувствительного узла [Вт/K].

Выражение для определения разности температур $\Delta T$ между приемной площадкой и ,холодными спаями“ используется для оценки верхнего предела измеряемой мощности:

$$
\Delta T=\varepsilon \cdot P / Q_{\Sigma},
$$

где $P$ - мощность оптического излучения, падающая на приемную площадку $[\mathrm{BT}]$. В данной работе под верхним 


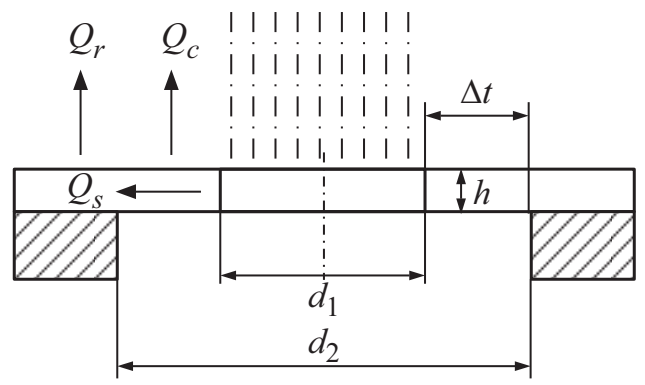

Pис. 1. Схема термоэлектрического приемника излучения.

пределом измеряемой оптической мощности подразумевалась величина, при которой перегрев составит $100^{\circ} \mathrm{C}$.

Постоянная времени $\tau$ [c] обусловливает быстродействие приемника:

$$
\tau=C / Q_{\Sigma},
$$

где $C$ - суммарная теплоемкость элементов, входящих в приемный узел приемника [Дж/К].

Из приведенных формул видно, что для повышения $R$ необходимо уменьшать $Q_{\Sigma}$, а также применять термопары с высокими значениями $\alpha$. Можно также повышать $R$, увеличивая число термопар, но следует заметить, что чрезмерное их увеличение может увеличить $Q_{\Sigma}$, общую теплоемкость и электрическое сопротивление, что приведет к ухудшению характеристик приемника. Поэтому конструкция чувствительного узла приемника должна быть оптимальной. С этой целью рассмотрим подробнее составляющие суммарной тепловой проводимости. В общем случае $Q_{\Sigma}$ является суммой тепловых проводимостей, представляющих различные механизмы прохождения тепловых потоков от приемного элемента в окружающую среду:

$$
Q_{\Sigma}=Q_{s}+Q_{r}+Q_{c}
$$

где $Q_{s}$ - тепловая проводимость по материалу подложки, $Q_{r}$ - тепловая проводимость за счет излучения, $Q_{c}$ - тепловая проводимость за счет конвекции.

Каждая из этих составляющих зависит от конкретной конструкции чувствительного узла, свойств используемых материалов, конструкции корпуса и ряда других факторов, поэтому рассмотрим схему устройства чувствительного узла, которая приведена на рис. 1.

На подложке, имеющей форму диска диаметром $d_{2}$, имеется приемная область в виде круга диаметром $d_{1}$, на котором располагается покрытие, обеспечивающее работу приемника в требуемом спектральном диапазоне. По наружному диаметру приемной области расположены тонкопленочные термопары, соединенные последовательно. Наружная область подложки расположена на массивном металлическом кольце, выполняющем функцию термостата.

Тепловая проводимость по материалу подложки $Q_{s}$ для устройства, приведенного на рис. 1, определяется из выражения

$$
Q_{s}=2 \pi \lambda h\left(\ln d_{2}-\ln d_{1}\right)
$$

где $d_{1}$ - диаметр приемной площадки, $d_{2}$ - диаметр подложки, $h$ - толщина подложки, $\lambda-$ удельная теплопроводность материала подложки.

Оценку тепловой проводимости $Q_{r}$ для небольших перепадов температур между нагретым телом и окружающей средой за счет излучения проведем по следующему выражению:

$$
Q_{r}=4 S \sigma \varepsilon T^{3}=2.2 \cdot 10^{-3} \mathrm{BT} / \mathrm{K},
$$

где $S$ - площадь поверхности приемного диска $\left[\mathrm{M}^{2}\right]$, $\sigma$ - постоянная Стефана-Больцмана, $T$ - температура окружающей среды, в данном случае принятая $T=303 \mathrm{~K}$. Примем коэффициент поглощения $\varepsilon=1$ (для обеих сторон диска). В выражении (6) используется удвоенное значение площади, так как обратная сторона диска так же излучает.

При заданных значениях температур на поверхности приемной площадки и окружающей среды определение $Q_{c}$ сводится к определению коэффициента теплоотдачи $\Lambda$. Вычисление среднего коэффициента теплоотдачи при свободном движении воздуха возможно с использованием чисел подобия по известным методикам [42-44]. В решаемой задаче для диаметра приемной площадки $15 \mathrm{Mм}$ и ее температуре $30^{\circ} \mathrm{C}$, температуре

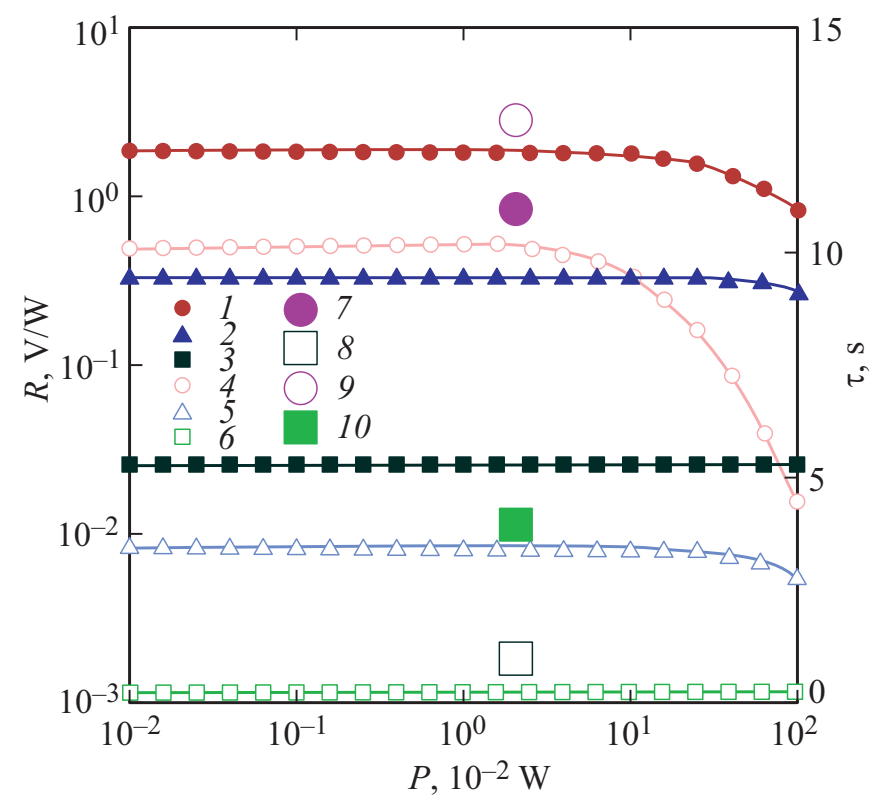

Рис. 2. Зависимости коэффициента преобразования $R$ и постоянной времени $\tau$ от поступающей мощности $P$. 1,2, 3 расчетные значения $R$ для подложек из полиимида, $\mathrm{SiO}_{2}$ и $\mathrm{AlN}$ соответственно; 4, 5, 6 - расчетные значения $\tau$ для подложек из полиимида, $\mathrm{SiO}_{2}$ и $\mathrm{AlN}$ соответственно; 7,10 - экспериментальные значения $R$ для подложек из полиимида и $\mathrm{AlN} ; 8,9$ - экспериментальные значения $\tau$ для подложек из полиимида и AlN. 

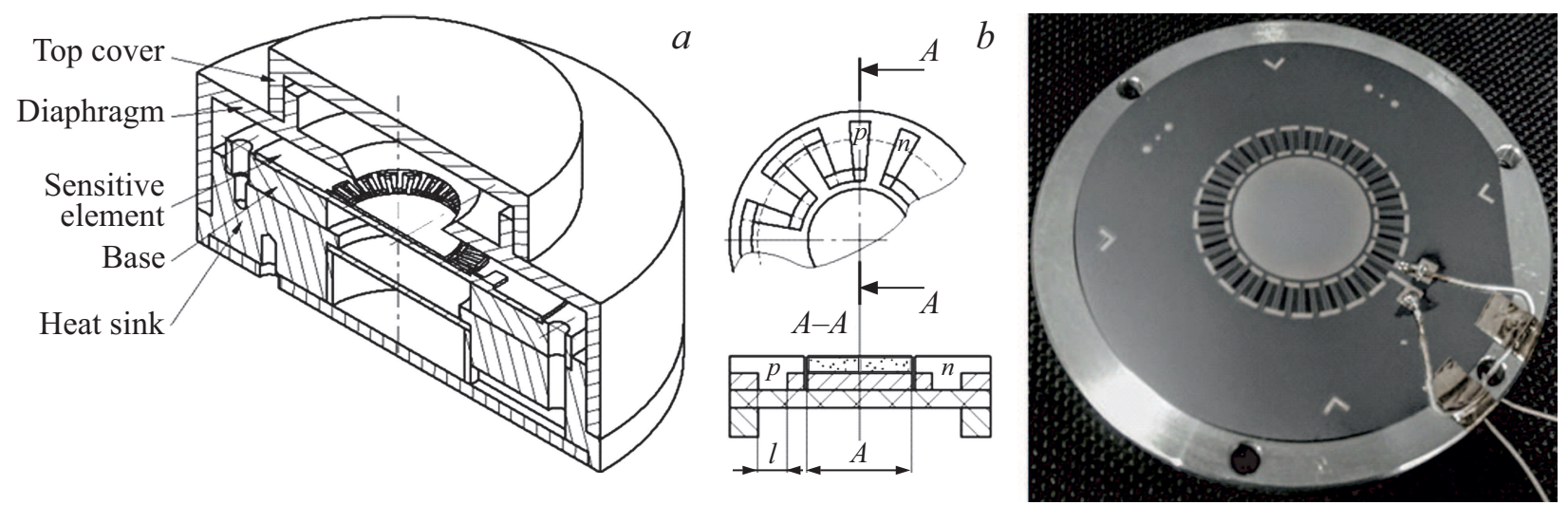

Рис. 3. Макет дискового приемника излучения: $a-$ схема конструкции макета дискового приемника излучения; $b-$ схема многоэлементной топологии макета дискового приемника излучения; $c$ - фотография термоэлемента макета дискового приемника излучения.

окружающего воздуха $20^{\circ} \mathrm{C}$, кинематическом коэффициенте вязкости воздуха $2.59 \cdot 10^{-2} \mathrm{BT} / \mathrm{M} \cdot \mathrm{K}$ средний коэффициент теплоотдачи составил $19 \mathrm{BT} / \mathrm{M}^{2} \cdot \mathrm{K}$, а выражение для $Q_{c}$ имеет вид

$$
Q_{c}=\Lambda S=3.4 \cdot 10^{-3} \mathrm{BT} / \mathrm{K},
$$

На основе приведенных выражений рассчитаны значения $R$ и $\tau$ для термоэлектрических приемников излучения, состоящих из 30 термопар длиной 4 мм, с коэффициентом термоэдс в $400 \mathrm{MKB} / \mathrm{K}$, для случаев использования различных материалов подложек: полиимидных, $\mathrm{SiO}_{2}$ и AlN. Результаты расчета представлены графически на (рис. 2). Как видно из результатов расчета, при выбранных материалах и их толщинах величины коэффициентов преобразования максимально отличаются в 300 раз.

\section{3. Экспериментальная часть}

Для получения тонких пленок был использован метод импульсного лазерного осаждения, где источником из-

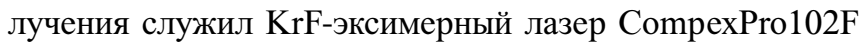
с длительностью импульса 30 нс и длиной волны 248 нм. Осаждение пленок осуществлялось с нагревом подложек и использованием защитного газа (Ar 99.99\%) при плотностях энергии лазерного излучения 1.5 Дж/ $\mathrm{cm}^{2}$. Для обеспечения однородности слоев использовалось вращение мишени и подложки, а также движение луча по поверхности мишени.

В качестве мишеней использовались цилиндрические монокристаллические слитки $\mathrm{Bi}_{0.5} \mathrm{Sb}_{1.5} \mathrm{Te}_{3}$ и $\mathrm{Bi}_{2} \mathrm{Te}_{2.7} \mathrm{Se}_{0.3}$. Согласно паспортным данным, материалы $p$ - и $n$-типа обладают коэффициентом Зеебека $205 \pm 5$ и $200 \pm 10 \mathrm{мкB} \cdot \mathrm{K}^{-1}$, электропроводностью $1050 \pm 150$ и $950 \pm 150 \mathrm{OM}^{-1} \cdot \mathrm{cm}^{-1}$ соответственно и удельной теплопроводностью около $1.4 \pm 0.05 \mathrm{BT} \cdot \mathrm{M}^{-1} \cdot \mathrm{K}^{-1}$. В качестве подложек использовался полиимидный материал, аналогичный Kapton, толщиной 100 мкм и подложки из AlN толщиной 250 мкм.

Топологии чувствительных элементов создавались масочным методом в дисковой геометрии. Конструкция включает в себя основание чувствительного элемента, чувствительный элемент, радиатор, диафрагму, переднюю крышку и заднюю крышку (рис. 3, $a$ ). Чувствительный элемент представляет собой подложку с нанесенными на нее термоэлектрическими, адгезионными и коммутационными слоями. Термоэлектрические дорожки образуют замкнутый контур, а в центре располагается приемная площадка (рис. $3, b$ и $c$ ). Приемные площадки изготовленных приемников были покрыты акриловой черной матовой эмалью, имеющей коэффициент поглощения не хуже $(95 \pm 3) \%$ в широкой области спектра.

Оценка коэффициента преобразования макетов приемников производилась по следующей методике: в плоскости расположения приемной площадки измеряемого приемника измерялась освещенность, создаваемая точечным источником света (лампа типа КГМ), рассчитывался поток $P$, приходящий на приемную площадку, измерялся сигнал $U_{C}$ с приемника и рассчитывался коэффициент преобразования по выражению

$$
R=U_{C} / P=U_{C} / E S,
$$

где $U_{C}-$ сигнал с приемника $[\mathrm{B}], E-$ освещенность в плоскости приемной площадки $\left[\mathrm{BT} / \mathrm{M}^{2}\right]$.

Сигнал с приемника измерялся вольтметром типа АРРА-09, освещенность измерялась образцовым приемником типа ПОИ-1 № 112003 г. с конусным приемным элементом. Данный приемник имеет обмотку нагревателя, позволяющую производить калибровку с помощью электрической мощности. Конструкция приемника обеспечивает высокую эквивалентность замещения оптической мощности электрической (не хуже $1 \%$ ). Основные характеристики образцового приемника: коэффициент преобразования 0.266 В/Вт, диаметр входной диафрагмы 
6 мм; площадь входной диафрагмы $28 \cdot 10^{-6} \mathrm{M}^{2}$. Измерения характеристики зонной однородности коэффициента преобразования приемной площадки проводились на стенде при автоматизированном перемещении измеряемых образцов и специально разработанной методике, учитывающей инерционные эффекты роста сигнала.

\section{4. Результаты и обсуждение}

Расчетные характеристики и результаты испытаний экспериментальных образцов показаны на (рис. 2). Из результатов расчета следует, что при использовании тонких материалов и материалов с малой теплопроводностью подложки потери на излучение могут быть сравнимы с потерями по подложке. Коэффициент преобразования выше всего при использовании полиимидных подложек в силу их низкой удельной теплопроводности $(0.14 \mathrm{BT} / \mathrm{M} \cdot \mathrm{K})$ по сравнению с оксидом кремния $(\sim 10 \mathrm{BT} / \mathrm{M} \cdot \mathrm{K})$ и нитридом алюминия $(\sim 150$ Вт/м $\cdot \mathrm{K})$. Коэффициенты преобразования, полученные для экспериментальных образцов с подложками из полиимида и нитрида алюминия, оказались близки к расчетным и составили 0.95 и $0.02 \mathrm{~B} / \mathrm{B}$ с соответственно. В силу различных теплофизических свойств из графика видно, что использование полиимидных оснований целесообразно при измерении потоков излучения мощностью $<100$ мВт.

Постоянные времени приемников, характеризующие их быстродействие, также сильно отличаются для различных материалов подложек и составляют $0.1,3$ и 10 с для $\mathrm{AlN}, \mathrm{SiO}_{2}$ и полиимидного материалов соответственно. Экспериментальные значения 12 и $0.5 \mathrm{c}$, полученные для сенсоров с полиимидным и $\mathrm{AlN}$ основанием соответственно, близки с расчетным и отличаются в силу принятых при расчете допущений.

Дополнительно проводились исследования зонной характеристики коэффициента чувствительности макетно-

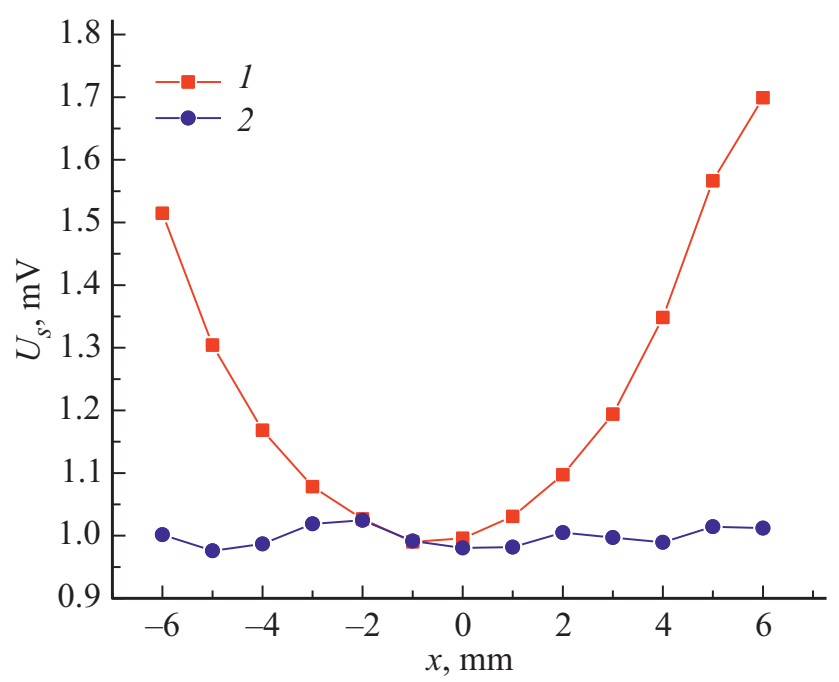

Pис. 4. График зонной характеристики образцов термоэлектрических сенсоров: 1 - до модификации приемной площадки, 2 - после модификации приемной площадки. го образца приемника излучения, выполненного на полиимидной подложке. Как видно из рис. 4, $a$, полиимидное основание обладает недостаточными для обеспечения высокой однородности тепловыми свойствами, поэтому тепловая масса приемной площадки была увеличена с использованием слоя алюминия, что позволило снизить погрешность зонной неоднородности до $1.0 \%$ (рис. $4, b$ ). Подложки из нитрида алюминия обеспечивают высокую линейность коэффициента преобразования в диапазоне мощностей до 1 Вт, обладают быстродействием $\sim 1 \mathrm{c}$, что также хорошо сходится с результатами теплового моделирования.

Таким образом, при создании высокочувствительных сенсоров с большой апертурой, использующих полиимидное основание, необходимо дополнительное увеличение тепловой массы приемной площадки с целью снижения погрешности зонной неоднородности. В случаях массивных теплопроводящих подложек AlN увеличения тепловой массы может не потребоваться.

\section{5. Заключение}

В данной работе проведено теоретическое и экспериментальное исследование перспективности использования радиационных термоэлементов, выполненных из тонких пленок $p$ - $\mathrm{Bi}_{0.5} \mathrm{Sb}_{1.5} \mathrm{Te}_{3}$ и $n-\mathrm{Bi}_{2} \mathrm{Te}_{2.7} \mathrm{Se}_{0.3}$ на различных подложках. Выбранный расчетный метод коэффициента преобразования и постоянной времени изделий показал высокую сходимость с результатами исследований опытных образцов.

В работе показано, что, применяя диски из материалов с различными коэффициентами теплопроводности, можно в широких диапазонах изменять основные характеристики приемника, такие как коэффициент преобразования и постоянную времени. Это позволяет, не меняя отработанную технологию осаждения тонкопленочных слоев термопар, не меняя трудоемкой в изготовлении оснастки, не меняя конструкции приемного узла, создать довольно широкий спектр приемников оптического излучения в качестве рабочих средств измерения (РСИ), способных измерять мощности от единиц мкВт до 10 ВТ без особых изменений конструкции термостата.

Для больших мощностей излучения потребуется дополнительное охлаждение термостата. Принудительное воздушное охлаждение целесообразно для мощностей от 10 до 100 Вт. Для измерения больших (до 1000 Вт) мощностей потребуется использование материалов, работающих при высоких температурах, и водяное охлаждение.

\section{Список литературы}

[1] F. Völklein. Sensors Actuators A: Physical, 29 (2), 87 (1991).

[2] J. Schieferdecker. Sensors Actuators A: Physical, 47 (1-3), 422 (1995).

[3] J. Lerchner. J. Microbiological Methods, 74 (2-3), 74 (2008). 
[4] W. Lee, W. Fon, B.W. Axelrod, M.L. Roukes. Proc. Natl. Acad. Sci. USA, 106 (36), 15225 (2009).

[5] V.L. Kopparthy. Bioengineering, 2, 2 (2015).

[6] R. Frank. Sensors Actuators B, 123, 413 (2007).

[7] S-C. Park. Analyst, 2 (134), 236 (2009).

[8] E.M. Vieira. J. Alloys Comp., 774 (5), 1102 (2019).

[9] R. Buchner. J. Microelectromechanical Systems, 17 (5), 1114 (2008).

[10] J.H. Lehman. Appl. Optics, 50 (21), 4099 (2011).

[11] W. Smetana. Sensors Actuators A: Physical, 37-38, 565 (1993).

[12] Y.Y. Protasov. Pribory i Tekhnika Eksperimenta, 45 (6), 70 (2002).

[13] V.V. Ryzhkov. Materials Today: Proceedings, 5(4), 10371 (2018).

[14] N.A. Djuzhev, D.Y. Novikov, G.D. Demin, A.I. Ovodov, V.T. Ryabov. IEEE Sensors Appl. Symp. (2018).

[15] A.G. Volkov. Cosmic Research, 55 (2), 124 (2017).

[16] M. Grott. Sci. Reviews, 208 (1-4), 413 (2017).

[17] R.A. Poshekhonov. Semiconductors, 51 (8), 981 (2017).

[18] A.S. Osipkov. J. Electron. Mater., 46 (10), 6195 (2017).

[19] G.J. Snyder. Nature, 7, 105 (2008).

[20] Б.М. Гольцман, В.А. Кудинов, И.А. Смирнов. Полупроводниковые термоэлектрические материалы на основе $\mathrm{Bi}_{2} \mathrm{Te}_{3}$ (М., Наука, 1972).

[21] L.M. Goncalves. Thin Sol. Films, 518, 2816 (2010).

[22] B. Huang. J. Appl. Phys., 104, 113710 (2008).

[23] H-J. Lee. Electron. Mater. Lett., 7 (1), 45 (2011).

[24] Y. Zhou. J. Alloys Comp., 590, 362 (2014).

[25] N. Peranio. J. Alloys Comp., 521, 163 (2012).

[26] J. Krumrain. J. Cryst. Growth, 324 (1), 115 (2011).

[27] H. Böttner, G. Chen, R. Venkatasubramanian. MRS Bulletin, 31, 211 (2006).

[28] H. Cao. Appl. Phys. Lett., 101, 162104 (2012).

[29] A. Boulouz. J. Materials, 2014, 8 (2014).

[30] L.M. Goncalves. Sensors Actuators A, 130-131, 346 (2006).

[31] P.H. Le. J. Alloys Comp., 615, 546 (2014).

[32] M. Ohta. Mater. Transactions, 50 (9), 2129 (2009).

[33] T. Sun. J. Cryst. Growth, 311 (16), 4123 (2009).

[34] E. Symeou. Appl. Surf. Sci., 336, 138 (2015)

[35] J. Schou. Appl. Surf. Sci., 255, 5191 (2009).

[36] P. R. Willmott. Rev. Mod. Phys., 72 (1), 315 (2000).

[37] Л.И. Анатычук. Термоэлектрические преобразователи энергии (Букрек, Киев, Черновцы, Украина, 2003).

[38] Б.М. Гольцман, 3.М. Дашевский, В.И. Кайданов, Н.В. Коломоец. Пленочные термоэлементы: физика и применение (М., Наука, 1985).

[39] В.П. Михеев, А.В. Просандеев. Датчики и детекторы (М., МИФИ, 2007).

[40] U. Dillner. J. Sensors and Sensor Systems, 2, 85 (2013).

[41] R.A. Mironov. Int. J. Heat and Mass Transfer, 127, 1230 (2018).

[42] Е.А. Краснощеков, А.С. Сукомел. Задачник по теплопередаче: Учеб. пособие для вузов (М., Энергия, 1980).

[43] М.А. Михеев, И.М. Михеева. Основы теплопередачи (М., Энергия, 1977).

[44] Д.В. Сивухин. Термодинамика и молекулярнал физика (М., Физматлит, 2005).

\section{Bismuth telluride based radiation thermopiles obtained by pulsed laser deposition method}

\author{
A.E. Shupenev ${ }^{1}$, I.S. Korshunov ${ }^{1}$, A.S. Iliin ${ }^{2}$, \\ A.S. Osipkov ${ }^{1}$, A.G. Grigoryants ${ }^{1}$ \\ ${ }^{1}$ Bauman Moscow State Technical University, \\ 105005 Moscow, Russia \\ 2 Joint Institute for High Temperatures (JIHT), \\ Russian Academy of Sciences, \\ 125412 Moscow, Russia
}

\begin{abstract}
Radiation thermopiles are basic structures for sensors used for measuring energy parameters of different radiation with wavelength from 0.1 to $100 \mathrm{mcm}$. Current work describes features of using $p$ - $\mathrm{Bi}_{0.5} \mathrm{Sb}_{1.5} \mathrm{Te}_{3}$ and $n-\mathrm{Bi}_{2} \mathrm{Te}_{2.7} \mathrm{Se}_{0.3}$ thin film thermopiles obtained with pulsed laser deposition method on different substrates. Thermal modeling and experimental research reveal the possibility of achieving responsivity of $1 \mathrm{~V} / \mathrm{W}$ and time constant of 10 seconds with the use of polyimide substrates and absorption zone of $16 \mathrm{~mm}$ in diameter.
\end{abstract}

